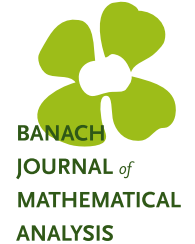

Banach J. Math. Anal. 10 (2016), no. 4, 783-799

http://dx.doi.org/10.1215/17358787-3649458

ISSN: $1735-8787$ (electronic)

http://projecteuclid.org/bjma

\title{
COVER-STRICT TOPOLOGIES, IDEALS, AND QUOTIENTS FOR SOME SPACES OF VECTOR-VALUED FUNCTIONS
}

\author{
TERJE HÕIM ${ }^{1}$ and D. A. ROBBINS $2^{*}$ \\ Communicated by N. Grønbæk
}

\begin{abstract}
Let $X$ be a completely regular Hausdorff space, let $\mathcal{D}$ be a cover of $X$, and let $\pi: \mathcal{E} \rightarrow X$ be a bundle of Banach spaces (algebras). Let $\Gamma(\pi)$ be the space of sections of $\pi$, and let $\Gamma_{b}(\pi, \mathcal{D})$ be the subspace of $\Gamma(\pi)$ consisting of sections which are bounded on each $D \in \mathcal{D}$. We study the subspace (ideal) and quotient structures of some spaces of vector-valued functions which arise from endowing $\Gamma_{b}(\pi, \mathcal{D})$ with the cover-strict topology.
\end{abstract}

\section{INTRODUCTION}

The present article investigates the ideal and quotient structures of certain algebras of vector-valued functions. By using the theory of bundles of topological vector spaces, our results extend to more general algebras many of the results to be found in [1] and [3] regarding the structure of some ideals and quotients of $C(X)$, where $X$ is a completely regular Hausdorff space.

We will be concerned with certain subspaces and quotients of $\Gamma(\pi)$, the space of sections of the bundle of Banach spaces (i.e., Banach bundle) $\pi: \mathcal{E} \rightarrow X$, and in particular, we will investigate such structures when $\pi: \mathcal{E} \rightarrow X$ is a bundle of Banach algebras (i.e., Banach algebra bundle). (For details of the development of such bundles, and of bundles of topological vector spaces in general, we refer the reader to [5]; further elaboration can be found in [10], [12], [6], and [7].) The essentials are the following (they can be found, e.g., in [7]), but we repeat them here for convenience. Our paper is also related to the theory of approximation

Copyright 2016 by the Tusi Mathematical Research Group.

Received Aug. 10, 2015; Accepted Jan. 18, 2016.

*Corresponding author.

2010 Mathematics Subject Classification. Primary 46H25; Secondary 46H10.

Keywords. cover-strict topology, bundle of Banach spaces, bundle of Banach algebras. 


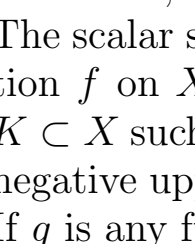

Banach J. Math. Anal. 10 (2016), no. 4, 783-799

http://dx.doi.org/10.1215/17358787-3649458

ISSN: $1735-8787$ (electronic)

http://projecteuclid.org/bjma

\title{
COVER-STRICT TOPOLOGIES, IDEALS, AND QUOTIENTS FOR SOME SPACES OF VECTOR-VALUED FUNCTIONS
}

\author{
TERJE HÕIM ${ }^{1}$ and D. A. ROBBINS $2^{*}$ \\ Communicated by N. Grønbæk
}

\begin{abstract}
Let $X$ be a completely regular Hausdorff space, let $\mathcal{D}$ be a cover of $X$, and let $\pi: \mathcal{E} \rightarrow X$ be a bundle of Banach spaces (algebras). Let $\Gamma(\pi)$ be the space of sections of $\pi$, and let $\Gamma_{b}(\pi, \mathcal{D})$ be the subspace of $\Gamma(\pi)$ consisting of sections which are bounded on each $D \in \mathcal{D}$. We study the subspace (ideal) and quotient structures of some spaces of vector-valued functions which arise from endowing $\Gamma_{b}(\pi, \mathcal{D})$ with the cover-strict topology.
\end{abstract}

\section{INTRODUCTION}

The present article investigates the ideal and quotient structures of certain algebras of vector-valued functions. By using the theory of bundles of topological vector spaces, our results extend to more general algebras many of the results to be found in [1] and [3] regarding the structure of some ideals and quotients of $C(X)$, where $X$ is a completely regular Hausdorff space.

We will be concerned with certain subspaces and quotients of $\Gamma(\pi)$, the space of sections of the bundle of Banach spaces (i.e., Banach bundle) $\pi: \mathcal{E} \rightarrow X$, and in particular, we will investigate such structures when $\pi: \mathcal{E} \rightarrow X$ is a bundle of Banach algebras (i.e., Banach algebra bundle). (For details of the development of such bundles, and of bundles of topological vector spaces in general, we refer the reader to [5]; further elaboration can be found in [10], [12], [6], and [7].) The essentials are the following (they can be found, e.g., in [7]), but we repeat them here for convenience. Our paper is also related to the theory of approximation

Copyright 2016 by the Tusi Mathematical Research Group.

Received Aug. 10, 2015; Accepted Jan. 18, 2016.

*Corresponding author.

2010 Mathematics Subject Classification. Primary 46H25; Secondary 46H10.

Keywords. cover-strict topology, bundle of Banach spaces, bundle of Banach algebras. 


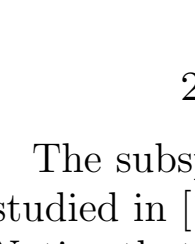

Banach J. Math. Anal. 10 (2016), no. 4, 783-799

http://dx.doi.org/10.1215/17358787-3649458

ISSN: $1735-8787$ (electronic)

http://projecteuclid.org/bjma

\title{
COVER-STRICT TOPOLOGIES, IDEALS, AND QUOTIENTS FOR SOME SPACES OF VECTOR-VALUED FUNCTIONS
}

\author{
TERJE HÕIM ${ }^{1}$ and D. A. ROBBINS $2^{*}$ \\ Communicated by N. Grønbæk
}

\begin{abstract}
Let $X$ be a completely regular Hausdorff space, let $\mathcal{D}$ be a cover of $X$, and let $\pi: \mathcal{E} \rightarrow X$ be a bundle of Banach spaces (algebras). Let $\Gamma(\pi)$ be the space of sections of $\pi$, and let $\Gamma_{b}(\pi, \mathcal{D})$ be the subspace of $\Gamma(\pi)$ consisting of sections which are bounded on each $D \in \mathcal{D}$. We study the subspace (ideal) and quotient structures of some spaces of vector-valued functions which arise from endowing $\Gamma_{b}(\pi, \mathcal{D})$ with the cover-strict topology.
\end{abstract}

\section{INTRODUCTION}

The present article investigates the ideal and quotient structures of certain algebras of vector-valued functions. By using the theory of bundles of topological vector spaces, our results extend to more general algebras many of the results to be found in [1] and [3] regarding the structure of some ideals and quotients of $C(X)$, where $X$ is a completely regular Hausdorff space.

We will be concerned with certain subspaces and quotients of $\Gamma(\pi)$, the space of sections of the bundle of Banach spaces (i.e., Banach bundle) $\pi: \mathcal{E} \rightarrow X$, and in particular, we will investigate such structures when $\pi: \mathcal{E} \rightarrow X$ is a bundle of Banach algebras (i.e., Banach algebra bundle). (For details of the development of such bundles, and of bundles of topological vector spaces in general, we refer the reader to [5]; further elaboration can be found in [10], [12], [6], and [7].) The essentials are the following (they can be found, e.g., in [7]), but we repeat them here for convenience. Our paper is also related to the theory of approximation

Copyright 2016 by the Tusi Mathematical Research Group.

Received Aug. 10, 2015; Accepted Jan. 18, 2016.

*Corresponding author.

2010 Mathematics Subject Classification. Primary 46H25; Secondary 46H10.

Keywords. cover-strict topology, bundle of Banach spaces, bundle of Banach algebras. 


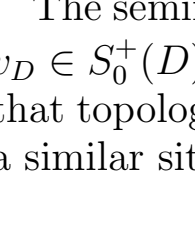

Banach J. Math. Anal. 10 (2016), no. 4, 783-799

http://dx.doi.org/10.1215/17358787-3649458

ISSN: $1735-8787$ (electronic)

http://projecteuclid.org/bjma

\title{
COVER-STRICT TOPOLOGIES, IDEALS, AND QUOTIENTS FOR SOME SPACES OF VECTOR-VALUED FUNCTIONS
}

\author{
TERJE HÕIM ${ }^{1}$ and D. A. ROBBINS $2^{*}$ \\ Communicated by N. Grønbæk
}

\begin{abstract}
Let $X$ be a completely regular Hausdorff space, let $\mathcal{D}$ be a cover of $X$, and let $\pi: \mathcal{E} \rightarrow X$ be a bundle of Banach spaces (algebras). Let $\Gamma(\pi)$ be the space of sections of $\pi$, and let $\Gamma_{b}(\pi, \mathcal{D})$ be the subspace of $\Gamma(\pi)$ consisting of sections which are bounded on each $D \in \mathcal{D}$. We study the subspace (ideal) and quotient structures of some spaces of vector-valued functions which arise from endowing $\Gamma_{b}(\pi, \mathcal{D})$ with the cover-strict topology.
\end{abstract}

\section{INTRODUCTION}

The present article investigates the ideal and quotient structures of certain algebras of vector-valued functions. By using the theory of bundles of topological vector spaces, our results extend to more general algebras many of the results to be found in [1] and [3] regarding the structure of some ideals and quotients of $C(X)$, where $X$ is a completely regular Hausdorff space.

We will be concerned with certain subspaces and quotients of $\Gamma(\pi)$, the space of sections of the bundle of Banach spaces (i.e., Banach bundle) $\pi: \mathcal{E} \rightarrow X$, and in particular, we will investigate such structures when $\pi: \mathcal{E} \rightarrow X$ is a bundle of Banach algebras (i.e., Banach algebra bundle). (For details of the development of such bundles, and of bundles of topological vector spaces in general, we refer the reader to [5]; further elaboration can be found in [10], [12], [6], and [7].) The essentials are the following (they can be found, e.g., in [7]), but we repeat them here for convenience. Our paper is also related to the theory of approximation

Copyright 2016 by the Tusi Mathematical Research Group.

Received Aug. 10, 2015; Accepted Jan. 18, 2016.

*Corresponding author.

2010 Mathematics Subject Classification. Primary 46H25; Secondary 46H10.

Keywords. cover-strict topology, bundle of Banach spaces, bundle of Banach algebras. 


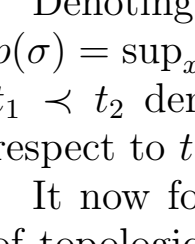

Banach J. Math. Anal. 10 (2016), no. 4, 783-799

http://dx.doi.org/10.1215/17358787-3649458

ISSN: $1735-8787$ (electronic)

http://projecteuclid.org/bjma

\title{
COVER-STRICT TOPOLOGIES, IDEALS, AND QUOTIENTS FOR SOME SPACES OF VECTOR-VALUED FUNCTIONS
}

\author{
TERJE HÕIM ${ }^{1}$ and D. A. ROBBINS $2^{*}$ \\ Communicated by N. Grønbæk
}

\begin{abstract}
Let $X$ be a completely regular Hausdorff space, let $\mathcal{D}$ be a cover of $X$, and let $\pi: \mathcal{E} \rightarrow X$ be a bundle of Banach spaces (algebras). Let $\Gamma(\pi)$ be the space of sections of $\pi$, and let $\Gamma_{b}(\pi, \mathcal{D})$ be the subspace of $\Gamma(\pi)$ consisting of sections which are bounded on each $D \in \mathcal{D}$. We study the subspace (ideal) and quotient structures of some spaces of vector-valued functions which arise from endowing $\Gamma_{b}(\pi, \mathcal{D})$ with the cover-strict topology.
\end{abstract}

\section{INTRODUCTION}

The present article investigates the ideal and quotient structures of certain algebras of vector-valued functions. By using the theory of bundles of topological vector spaces, our results extend to more general algebras many of the results to be found in [1] and [3] regarding the structure of some ideals and quotients of $C(X)$, where $X$ is a completely regular Hausdorff space.

We will be concerned with certain subspaces and quotients of $\Gamma(\pi)$, the space of sections of the bundle of Banach spaces (i.e., Banach bundle) $\pi: \mathcal{E} \rightarrow X$, and in particular, we will investigate such structures when $\pi: \mathcal{E} \rightarrow X$ is a bundle of Banach algebras (i.e., Banach algebra bundle). (For details of the development of such bundles, and of bundles of topological vector spaces in general, we refer the reader to [5]; further elaboration can be found in [10], [12], [6], and [7].) The essentials are the following (they can be found, e.g., in [7]), but we repeat them here for convenience. Our paper is also related to the theory of approximation

Copyright 2016 by the Tusi Mathematical Research Group.

Received Aug. 10, 2015; Accepted Jan. 18, 2016.

*Corresponding author.

2010 Mathematics Subject Classification. Primary 46H25; Secondary 46H10.

Keywords. cover-strict topology, bundle of Banach spaces, bundle of Banach algebras. 


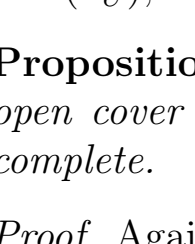

Banach J. Math. Anal. 10 (2016), no. 4, 783-799

http://dx.doi.org/10.1215/17358787-3649458

ISSN: $1735-8787$ (electronic)

http://projecteuclid.org/bjma

\title{
COVER-STRICT TOPOLOGIES, IDEALS, AND QUOTIENTS FOR SOME SPACES OF VECTOR-VALUED FUNCTIONS
}

\author{
TERJE HÕIM ${ }^{1}$ and D. A. ROBBINS $2^{*}$ \\ Communicated by N. Grønbæk
}

\begin{abstract}
Let $X$ be a completely regular Hausdorff space, let $\mathcal{D}$ be a cover of $X$, and let $\pi: \mathcal{E} \rightarrow X$ be a bundle of Banach spaces (algebras). Let $\Gamma(\pi)$ be the space of sections of $\pi$, and let $\Gamma_{b}(\pi, \mathcal{D})$ be the subspace of $\Gamma(\pi)$ consisting of sections which are bounded on each $D \in \mathcal{D}$. We study the subspace (ideal) and quotient structures of some spaces of vector-valued functions which arise from endowing $\Gamma_{b}(\pi, \mathcal{D})$ with the cover-strict topology.
\end{abstract}

\section{INTRODUCTION}

The present article investigates the ideal and quotient structures of certain algebras of vector-valued functions. By using the theory of bundles of topological vector spaces, our results extend to more general algebras many of the results to be found in [1] and [3] regarding the structure of some ideals and quotients of $C(X)$, where $X$ is a completely regular Hausdorff space.

We will be concerned with certain subspaces and quotients of $\Gamma(\pi)$, the space of sections of the bundle of Banach spaces (i.e., Banach bundle) $\pi: \mathcal{E} \rightarrow X$, and in particular, we will investigate such structures when $\pi: \mathcal{E} \rightarrow X$ is a bundle of Banach algebras (i.e., Banach algebra bundle). (For details of the development of such bundles, and of bundles of topological vector spaces in general, we refer the reader to [5]; further elaboration can be found in [10], [12], [6], and [7].) The essentials are the following (they can be found, e.g., in [7]), but we repeat them here for convenience. Our paper is also related to the theory of approximation

Copyright 2016 by the Tusi Mathematical Research Group.

Received Aug. 10, 2015; Accepted Jan. 18, 2016.

*Corresponding author.

2010 Mathematics Subject Classification. Primary 46H25; Secondary 46H10.

Keywords. cover-strict topology, bundle of Banach spaces, bundle of Banach algebras. 


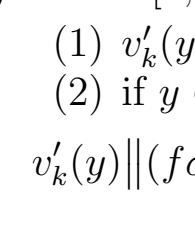

Banach J. Math. Anal. 10 (2016), no. 4, 783-799

http://dx.doi.org/10.1215/17358787-3649458

ISSN: $1735-8787$ (electronic)

http://projecteuclid.org/bjma

\title{
COVER-STRICT TOPOLOGIES, IDEALS, AND QUOTIENTS FOR SOME SPACES OF VECTOR-VALUED FUNCTIONS
}

\author{
TERJE HÕIM ${ }^{1}$ and D. A. ROBBINS $2^{*}$ \\ Communicated by N. Grønbæk
}

\begin{abstract}
Let $X$ be a completely regular Hausdorff space, let $\mathcal{D}$ be a cover of $X$, and let $\pi: \mathcal{E} \rightarrow X$ be a bundle of Banach spaces (algebras). Let $\Gamma(\pi)$ be the space of sections of $\pi$, and let $\Gamma_{b}(\pi, \mathcal{D})$ be the subspace of $\Gamma(\pi)$ consisting of sections which are bounded on each $D \in \mathcal{D}$. We study the subspace (ideal) and quotient structures of some spaces of vector-valued functions which arise from endowing $\Gamma_{b}(\pi, \mathcal{D})$ with the cover-strict topology.
\end{abstract}

\section{INTRODUCTION}

The present article investigates the ideal and quotient structures of certain algebras of vector-valued functions. By using the theory of bundles of topological vector spaces, our results extend to more general algebras many of the results to be found in [1] and [3] regarding the structure of some ideals and quotients of $C(X)$, where $X$ is a completely regular Hausdorff space.

We will be concerned with certain subspaces and quotients of $\Gamma(\pi)$, the space of sections of the bundle of Banach spaces (i.e., Banach bundle) $\pi: \mathcal{E} \rightarrow X$, and in particular, we will investigate such structures when $\pi: \mathcal{E} \rightarrow X$ is a bundle of Banach algebras (i.e., Banach algebra bundle). (For details of the development of such bundles, and of bundles of topological vector spaces in general, we refer the reader to [5]; further elaboration can be found in [10], [12], [6], and [7].) The essentials are the following (they can be found, e.g., in [7]), but we repeat them here for convenience. Our paper is also related to the theory of approximation

Copyright 2016 by the Tusi Mathematical Research Group.

Received Aug. 10, 2015; Accepted Jan. 18, 2016.

*Corresponding author.

2010 Mathematics Subject Classification. Primary 46H25; Secondary 46H10.

Keywords. cover-strict topology, bundle of Banach spaces, bundle of Banach algebras. 


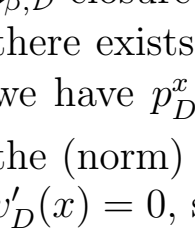

Banach J. Math. Anal. 10 (2016), no. 4, 783-799

http://dx.doi.org/10.1215/17358787-3649458

ISSN: $1735-8787$ (electronic)

http://projecteuclid.org/bjma

\title{
COVER-STRICT TOPOLOGIES, IDEALS, AND QUOTIENTS FOR SOME SPACES OF VECTOR-VALUED FUNCTIONS
}

\author{
TERJE HÕIM ${ }^{1}$ and D. A. ROBBINS $2^{*}$ \\ Communicated by N. Grønbæk
}

\begin{abstract}
Let $X$ be a completely regular Hausdorff space, let $\mathcal{D}$ be a cover of $X$, and let $\pi: \mathcal{E} \rightarrow X$ be a bundle of Banach spaces (algebras). Let $\Gamma(\pi)$ be the space of sections of $\pi$, and let $\Gamma_{b}(\pi, \mathcal{D})$ be the subspace of $\Gamma(\pi)$ consisting of sections which are bounded on each $D \in \mathcal{D}$. We study the subspace (ideal) and quotient structures of some spaces of vector-valued functions which arise from endowing $\Gamma_{b}(\pi, \mathcal{D})$ with the cover-strict topology.
\end{abstract}

\section{INTRODUCTION}

The present article investigates the ideal and quotient structures of certain algebras of vector-valued functions. By using the theory of bundles of topological vector spaces, our results extend to more general algebras many of the results to be found in [1] and [3] regarding the structure of some ideals and quotients of $C(X)$, where $X$ is a completely regular Hausdorff space.

We will be concerned with certain subspaces and quotients of $\Gamma(\pi)$, the space of sections of the bundle of Banach spaces (i.e., Banach bundle) $\pi: \mathcal{E} \rightarrow X$, and in particular, we will investigate such structures when $\pi: \mathcal{E} \rightarrow X$ is a bundle of Banach algebras (i.e., Banach algebra bundle). (For details of the development of such bundles, and of bundles of topological vector spaces in general, we refer the reader to [5]; further elaboration can be found in [10], [12], [6], and [7].) The essentials are the following (they can be found, e.g., in [7]), but we repeat them here for convenience. Our paper is also related to the theory of approximation

Copyright 2016 by the Tusi Mathematical Research Group.

Received Aug. 10, 2015; Accepted Jan. 18, 2016.

*Corresponding author.

2010 Mathematics Subject Classification. Primary 46H25; Secondary 46H10.

Keywords. cover-strict topology, bundle of Banach spaces, bundle of Banach algebras. 


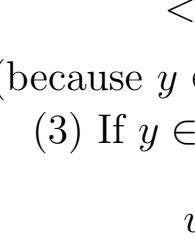

Banach J. Math. Anal. 10 (2016), no. 4, 783-799

http://dx.doi.org/10.1215/17358787-3649458

ISSN: $1735-8787$ (electronic)

http://projecteuclid.org/bjma

\title{
COVER-STRICT TOPOLOGIES, IDEALS, AND QUOTIENTS FOR SOME SPACES OF VECTOR-VALUED FUNCTIONS
}

\author{
TERJE HÕIM ${ }^{1}$ and D. A. ROBBINS $2^{*}$ \\ Communicated by N. Grønbæk
}

\begin{abstract}
Let $X$ be a completely regular Hausdorff space, let $\mathcal{D}$ be a cover of $X$, and let $\pi: \mathcal{E} \rightarrow X$ be a bundle of Banach spaces (algebras). Let $\Gamma(\pi)$ be the space of sections of $\pi$, and let $\Gamma_{b}(\pi, \mathcal{D})$ be the subspace of $\Gamma(\pi)$ consisting of sections which are bounded on each $D \in \mathcal{D}$. We study the subspace (ideal) and quotient structures of some spaces of vector-valued functions which arise from endowing $\Gamma_{b}(\pi, \mathcal{D})$ with the cover-strict topology.
\end{abstract}

\section{INTRODUCTION}

The present article investigates the ideal and quotient structures of certain algebras of vector-valued functions. By using the theory of bundles of topological vector spaces, our results extend to more general algebras many of the results to be found in [1] and [3] regarding the structure of some ideals and quotients of $C(X)$, where $X$ is a completely regular Hausdorff space.

We will be concerned with certain subspaces and quotients of $\Gamma(\pi)$, the space of sections of the bundle of Banach spaces (i.e., Banach bundle) $\pi: \mathcal{E} \rightarrow X$, and in particular, we will investigate such structures when $\pi: \mathcal{E} \rightarrow X$ is a bundle of Banach algebras (i.e., Banach algebra bundle). (For details of the development of such bundles, and of bundles of topological vector spaces in general, we refer the reader to [5]; further elaboration can be found in [10], [12], [6], and [7].) The essentials are the following (they can be found, e.g., in [7]), but we repeat them here for convenience. Our paper is also related to the theory of approximation

Copyright 2016 by the Tusi Mathematical Research Group.

Received Aug. 10, 2015; Accepted Jan. 18, 2016.

*Corresponding author.

2010 Mathematics Subject Classification. Primary 46H25; Secondary 46H10.

Keywords. cover-strict topology, bundle of Banach spaces, bundle of Banach algebras. 


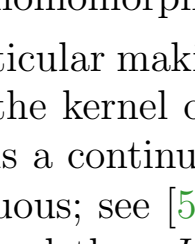

Banach J. Math. Anal. 10 (2016), no. 4, 783-799

http://dx.doi.org/10.1215/17358787-3649458

ISSN: $1735-8787$ (electronic)

http://projecteuclid.org/bjma

\title{
COVER-STRICT TOPOLOGIES, IDEALS, AND QUOTIENTS FOR SOME SPACES OF VECTOR-VALUED FUNCTIONS
}

\author{
TERJE HÕIM ${ }^{1}$ and D. A. ROBBINS $2^{*}$ \\ Communicated by N. Grønbæk
}

\begin{abstract}
Let $X$ be a completely regular Hausdorff space, let $\mathcal{D}$ be a cover of $X$, and let $\pi: \mathcal{E} \rightarrow X$ be a bundle of Banach spaces (algebras). Let $\Gamma(\pi)$ be the space of sections of $\pi$, and let $\Gamma_{b}(\pi, \mathcal{D})$ be the subspace of $\Gamma(\pi)$ consisting of sections which are bounded on each $D \in \mathcal{D}$. We study the subspace (ideal) and quotient structures of some spaces of vector-valued functions which arise from endowing $\Gamma_{b}(\pi, \mathcal{D})$ with the cover-strict topology.
\end{abstract}

\section{INTRODUCTION}

The present article investigates the ideal and quotient structures of certain algebras of vector-valued functions. By using the theory of bundles of topological vector spaces, our results extend to more general algebras many of the results to be found in [1] and [3] regarding the structure of some ideals and quotients of $C(X)$, where $X$ is a completely regular Hausdorff space.

We will be concerned with certain subspaces and quotients of $\Gamma(\pi)$, the space of sections of the bundle of Banach spaces (i.e., Banach bundle) $\pi: \mathcal{E} \rightarrow X$, and in particular, we will investigate such structures when $\pi: \mathcal{E} \rightarrow X$ is a bundle of Banach algebras (i.e., Banach algebra bundle). (For details of the development of such bundles, and of bundles of topological vector spaces in general, we refer the reader to [5]; further elaboration can be found in [10], [12], [6], and [7].) The essentials are the following (they can be found, e.g., in [7]), but we repeat them here for convenience. Our paper is also related to the theory of approximation

Copyright 2016 by the Tusi Mathematical Research Group.

Received Aug. 10, 2015; Accepted Jan. 18, 2016.

*Corresponding author.

2010 Mathematics Subject Classification. Primary 46H25; Secondary 46H10.

Keywords. cover-strict topology, bundle of Banach spaces, bundle of Banach algebras. 


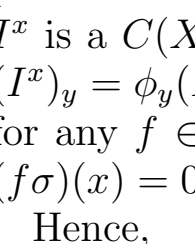

Banach J. Math. Anal. 10 (2016), no. 4, 783-799

http://dx.doi.org/10.1215/17358787-3649458

ISSN: $1735-8787$ (electronic)

http://projecteuclid.org/bjma

\title{
COVER-STRICT TOPOLOGIES, IDEALS, AND QUOTIENTS FOR SOME SPACES OF VECTOR-VALUED FUNCTIONS
}

\author{
TERJE HÕIM ${ }^{1}$ and D. A. ROBBINS $2^{*}$ \\ Communicated by N. Grønbæk
}

\begin{abstract}
Let $X$ be a completely regular Hausdorff space, let $\mathcal{D}$ be a cover of $X$, and let $\pi: \mathcal{E} \rightarrow X$ be a bundle of Banach spaces (algebras). Let $\Gamma(\pi)$ be the space of sections of $\pi$, and let $\Gamma_{b}(\pi, \mathcal{D})$ be the subspace of $\Gamma(\pi)$ consisting of sections which are bounded on each $D \in \mathcal{D}$. We study the subspace (ideal) and quotient structures of some spaces of vector-valued functions which arise from endowing $\Gamma_{b}(\pi, \mathcal{D})$ with the cover-strict topology.
\end{abstract}

\section{INTRODUCTION}

The present article investigates the ideal and quotient structures of certain algebras of vector-valued functions. By using the theory of bundles of topological vector spaces, our results extend to more general algebras many of the results to be found in [1] and [3] regarding the structure of some ideals and quotients of $C(X)$, where $X$ is a completely regular Hausdorff space.

We will be concerned with certain subspaces and quotients of $\Gamma(\pi)$, the space of sections of the bundle of Banach spaces (i.e., Banach bundle) $\pi: \mathcal{E} \rightarrow X$, and in particular, we will investigate such structures when $\pi: \mathcal{E} \rightarrow X$ is a bundle of Banach algebras (i.e., Banach algebra bundle). (For details of the development of such bundles, and of bundles of topological vector spaces in general, we refer the reader to [5]; further elaboration can be found in [10], [12], [6], and [7].) The essentials are the following (they can be found, e.g., in [7]), but we repeat them here for convenience. Our paper is also related to the theory of approximation

Copyright 2016 by the Tusi Mathematical Research Group.

Received Aug. 10, 2015; Accepted Jan. 18, 2016.

*Corresponding author.

2010 Mathematics Subject Classification. Primary 46H25; Secondary 46H10.

Keywords. cover-strict topology, bundle of Banach spaces, bundle of Banach algebras. 


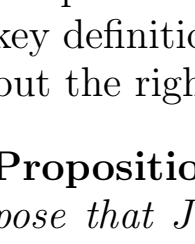

Banach J. Math. Anal. 10 (2016), no. 4, 783-799

http://dx.doi.org/10.1215/17358787-3649458

ISSN: $1735-8787$ (electronic)

http://projecteuclid.org/bjma

\title{
COVER-STRICT TOPOLOGIES, IDEALS, AND QUOTIENTS FOR SOME SPACES OF VECTOR-VALUED FUNCTIONS
}

\author{
TERJE HÕIM ${ }^{1}$ and D. A. ROBBINS $2^{*}$ \\ Communicated by N. Grønbæk
}

\begin{abstract}
Let $X$ be a completely regular Hausdorff space, let $\mathcal{D}$ be a cover of $X$, and let $\pi: \mathcal{E} \rightarrow X$ be a bundle of Banach spaces (algebras). Let $\Gamma(\pi)$ be the space of sections of $\pi$, and let $\Gamma_{b}(\pi, \mathcal{D})$ be the subspace of $\Gamma(\pi)$ consisting of sections which are bounded on each $D \in \mathcal{D}$. We study the subspace (ideal) and quotient structures of some spaces of vector-valued functions which arise from endowing $\Gamma_{b}(\pi, \mathcal{D})$ with the cover-strict topology.
\end{abstract}

\section{INTRODUCTION}

The present article investigates the ideal and quotient structures of certain algebras of vector-valued functions. By using the theory of bundles of topological vector spaces, our results extend to more general algebras many of the results to be found in [1] and [3] regarding the structure of some ideals and quotients of $C(X)$, where $X$ is a completely regular Hausdorff space.

We will be concerned with certain subspaces and quotients of $\Gamma(\pi)$, the space of sections of the bundle of Banach spaces (i.e., Banach bundle) $\pi: \mathcal{E} \rightarrow X$, and in particular, we will investigate such structures when $\pi: \mathcal{E} \rightarrow X$ is a bundle of Banach algebras (i.e., Banach algebra bundle). (For details of the development of such bundles, and of bundles of topological vector spaces in general, we refer the reader to [5]; further elaboration can be found in [10], [12], [6], and [7].) The essentials are the following (they can be found, e.g., in [7]), but we repeat them here for convenience. Our paper is also related to the theory of approximation

Copyright 2016 by the Tusi Mathematical Research Group.

Received Aug. 10, 2015; Accepted Jan. 18, 2016.

*Corresponding author.

2010 Mathematics Subject Classification. Primary 46H25; Secondary 46H10.

Keywords. cover-strict topology, bundle of Banach spaces, bundle of Banach algebras. 


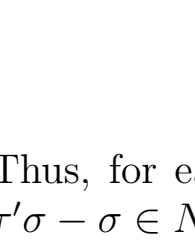

Banach J. Math. Anal. 10 (2016), no. 4, 783-799

http://dx.doi.org/10.1215/17358787-3649458

ISSN: $1735-8787$ (electronic)

http://projecteuclid.org/bjma

\title{
COVER-STRICT TOPOLOGIES, IDEALS, AND QUOTIENTS FOR SOME SPACES OF VECTOR-VALUED FUNCTIONS
}

\author{
TERJE HÕIM ${ }^{1}$ and D. A. ROBBINS $2^{*}$ \\ Communicated by N. Grønbæk
}

\begin{abstract}
Let $X$ be a completely regular Hausdorff space, let $\mathcal{D}$ be a cover of $X$, and let $\pi: \mathcal{E} \rightarrow X$ be a bundle of Banach spaces (algebras). Let $\Gamma(\pi)$ be the space of sections of $\pi$, and let $\Gamma_{b}(\pi, \mathcal{D})$ be the subspace of $\Gamma(\pi)$ consisting of sections which are bounded on each $D \in \mathcal{D}$. We study the subspace (ideal) and quotient structures of some spaces of vector-valued functions which arise from endowing $\Gamma_{b}(\pi, \mathcal{D})$ with the cover-strict topology.
\end{abstract}

\section{INTRODUCTION}

The present article investigates the ideal and quotient structures of certain algebras of vector-valued functions. By using the theory of bundles of topological vector spaces, our results extend to more general algebras many of the results to be found in [1] and [3] regarding the structure of some ideals and quotients of $C(X)$, where $X$ is a completely regular Hausdorff space.

We will be concerned with certain subspaces and quotients of $\Gamma(\pi)$, the space of sections of the bundle of Banach spaces (i.e., Banach bundle) $\pi: \mathcal{E} \rightarrow X$, and in particular, we will investigate such structures when $\pi: \mathcal{E} \rightarrow X$ is a bundle of Banach algebras (i.e., Banach algebra bundle). (For details of the development of such bundles, and of bundles of topological vector spaces in general, we refer the reader to [5]; further elaboration can be found in [10], [12], [6], and [7].) The essentials are the following (they can be found, e.g., in [7]), but we repeat them here for convenience. Our paper is also related to the theory of approximation

Copyright 2016 by the Tusi Mathematical Research Group.

Received Aug. 10, 2015; Accepted Jan. 18, 2016.

*Corresponding author.

2010 Mathematics Subject Classification. Primary 46H25; Secondary 46H10.

Keywords. cover-strict topology, bundle of Banach spaces, bundle of Banach algebras. 


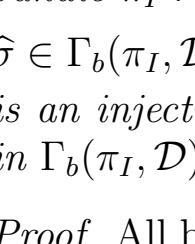

Banach J. Math. Anal. 10 (2016), no. 4, 783-799

http://dx.doi.org/10.1215/17358787-3649458

ISSN: $1735-8787$ (electronic)

http://projecteuclid.org/bjma

\title{
COVER-STRICT TOPOLOGIES, IDEALS, AND QUOTIENTS FOR SOME SPACES OF VECTOR-VALUED FUNCTIONS
}

\author{
TERJE HÕIM ${ }^{1}$ and D. A. ROBBINS $2^{*}$ \\ Communicated by N. Grønbæk
}

\begin{abstract}
Let $X$ be a completely regular Hausdorff space, let $\mathcal{D}$ be a cover of $X$, and let $\pi: \mathcal{E} \rightarrow X$ be a bundle of Banach spaces (algebras). Let $\Gamma(\pi)$ be the space of sections of $\pi$, and let $\Gamma_{b}(\pi, \mathcal{D})$ be the subspace of $\Gamma(\pi)$ consisting of sections which are bounded on each $D \in \mathcal{D}$. We study the subspace (ideal) and quotient structures of some spaces of vector-valued functions which arise from endowing $\Gamma_{b}(\pi, \mathcal{D})$ with the cover-strict topology.
\end{abstract}

\section{INTRODUCTION}

The present article investigates the ideal and quotient structures of certain algebras of vector-valued functions. By using the theory of bundles of topological vector spaces, our results extend to more general algebras many of the results to be found in [1] and [3] regarding the structure of some ideals and quotients of $C(X)$, where $X$ is a completely regular Hausdorff space.

We will be concerned with certain subspaces and quotients of $\Gamma(\pi)$, the space of sections of the bundle of Banach spaces (i.e., Banach bundle) $\pi: \mathcal{E} \rightarrow X$, and in particular, we will investigate such structures when $\pi: \mathcal{E} \rightarrow X$ is a bundle of Banach algebras (i.e., Banach algebra bundle). (For details of the development of such bundles, and of bundles of topological vector spaces in general, we refer the reader to [5]; further elaboration can be found in [10], [12], [6], and [7].) The essentials are the following (they can be found, e.g., in [7]), but we repeat them here for convenience. Our paper is also related to the theory of approximation

Copyright 2016 by the Tusi Mathematical Research Group.

Received Aug. 10, 2015; Accepted Jan. 18, 2016.

*Corresponding author.

2010 Mathematics Subject Classification. Primary 46H25; Secondary 46H10.

Keywords. cover-strict topology, bundle of Banach spaces, bundle of Banach algebras. 


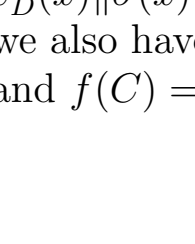

Banach J. Math. Anal. 10 (2016), no. 4, 783-799

http://dx.doi.org/10.1215/17358787-3649458

ISSN: $1735-8787$ (electronic)

http://projecteuclid.org/bjma

\title{
COVER-STRICT TOPOLOGIES, IDEALS, AND QUOTIENTS FOR SOME SPACES OF VECTOR-VALUED FUNCTIONS
}

\author{
TERJE HÕIM ${ }^{1}$ and D. A. ROBBINS $2^{*}$ \\ Communicated by N. Grønbæk
}

\begin{abstract}
Let $X$ be a completely regular Hausdorff space, let $\mathcal{D}$ be a cover of $X$, and let $\pi: \mathcal{E} \rightarrow X$ be a bundle of Banach spaces (algebras). Let $\Gamma(\pi)$ be the space of sections of $\pi$, and let $\Gamma_{b}(\pi, \mathcal{D})$ be the subspace of $\Gamma(\pi)$ consisting of sections which are bounded on each $D \in \mathcal{D}$. We study the subspace (ideal) and quotient structures of some spaces of vector-valued functions which arise from endowing $\Gamma_{b}(\pi, \mathcal{D})$ with the cover-strict topology.
\end{abstract}

\section{INTRODUCTION}

The present article investigates the ideal and quotient structures of certain algebras of vector-valued functions. By using the theory of bundles of topological vector spaces, our results extend to more general algebras many of the results to be found in [1] and [3] regarding the structure of some ideals and quotients of $C(X)$, where $X$ is a completely regular Hausdorff space.

We will be concerned with certain subspaces and quotients of $\Gamma(\pi)$, the space of sections of the bundle of Banach spaces (i.e., Banach bundle) $\pi: \mathcal{E} \rightarrow X$, and in particular, we will investigate such structures when $\pi: \mathcal{E} \rightarrow X$ is a bundle of Banach algebras (i.e., Banach algebra bundle). (For details of the development of such bundles, and of bundles of topological vector spaces in general, we refer the reader to [5]; further elaboration can be found in [10], [12], [6], and [7].) The essentials are the following (they can be found, e.g., in [7]), but we repeat them here for convenience. Our paper is also related to the theory of approximation

Copyright 2016 by the Tusi Mathematical Research Group.

Received Aug. 10, 2015; Accepted Jan. 18, 2016.

*Corresponding author.

2010 Mathematics Subject Classification. Primary 46H25; Secondary 46H10.

Keywords. cover-strict topology, bundle of Banach spaces, bundle of Banach algebras. 


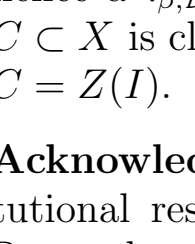

Banach J. Math. Anal. 10 (2016), no. 4, 783-799

http://dx.doi.org/10.1215/17358787-3649458

ISSN: $1735-8787$ (electronic)

http://projecteuclid.org/bjma

\title{
COVER-STRICT TOPOLOGIES, IDEALS, AND QUOTIENTS FOR SOME SPACES OF VECTOR-VALUED FUNCTIONS
}

\author{
TERJE HÕIM ${ }^{1}$ and D. A. ROBBINS $2^{*}$ \\ Communicated by N. Grønbæk
}

\begin{abstract}
Let $X$ be a completely regular Hausdorff space, let $\mathcal{D}$ be a cover of $X$, and let $\pi: \mathcal{E} \rightarrow X$ be a bundle of Banach spaces (algebras). Let $\Gamma(\pi)$ be the space of sections of $\pi$, and let $\Gamma_{b}(\pi, \mathcal{D})$ be the subspace of $\Gamma(\pi)$ consisting of sections which are bounded on each $D \in \mathcal{D}$. We study the subspace (ideal) and quotient structures of some spaces of vector-valued functions which arise from endowing $\Gamma_{b}(\pi, \mathcal{D})$ with the cover-strict topology.
\end{abstract}

\section{INTRODUCTION}

The present article investigates the ideal and quotient structures of certain algebras of vector-valued functions. By using the theory of bundles of topological vector spaces, our results extend to more general algebras many of the results to be found in [1] and [3] regarding the structure of some ideals and quotients of $C(X)$, where $X$ is a completely regular Hausdorff space.

We will be concerned with certain subspaces and quotients of $\Gamma(\pi)$, the space of sections of the bundle of Banach spaces (i.e., Banach bundle) $\pi: \mathcal{E} \rightarrow X$, and in particular, we will investigate such structures when $\pi: \mathcal{E} \rightarrow X$ is a bundle of Banach algebras (i.e., Banach algebra bundle). (For details of the development of such bundles, and of bundles of topological vector spaces in general, we refer the reader to [5]; further elaboration can be found in [10], [12], [6], and [7].) The essentials are the following (they can be found, e.g., in [7]), but we repeat them here for convenience. Our paper is also related to the theory of approximation

Copyright 2016 by the Tusi Mathematical Research Group.

Received Aug. 10, 2015; Accepted Jan. 18, 2016.

*Corresponding author.

2010 Mathematics Subject Classification. Primary 46H25; Secondary 46H10.

Keywords. cover-strict topology, bundle of Banach spaces, bundle of Banach algebras. 


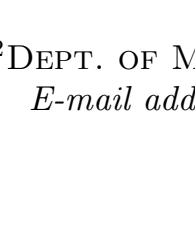

Banach J. Math. Anal. 10 (2016), no. 4, 783-799

http://dx.doi.org/10.1215/17358787-3649458

ISSN: $1735-8787$ (electronic)

http://projecteuclid.org/bjma

\title{
COVER-STRICT TOPOLOGIES, IDEALS, AND QUOTIENTS FOR SOME SPACES OF VECTOR-VALUED FUNCTIONS
}

\author{
TERJE HÕIM ${ }^{1}$ and D. A. ROBBINS $2^{*}$ \\ Communicated by N. Grønbæk
}

\begin{abstract}
Let $X$ be a completely regular Hausdorff space, let $\mathcal{D}$ be a cover of $X$, and let $\pi: \mathcal{E} \rightarrow X$ be a bundle of Banach spaces (algebras). Let $\Gamma(\pi)$ be the space of sections of $\pi$, and let $\Gamma_{b}(\pi, \mathcal{D})$ be the subspace of $\Gamma(\pi)$ consisting of sections which are bounded on each $D \in \mathcal{D}$. We study the subspace (ideal) and quotient structures of some spaces of vector-valued functions which arise from endowing $\Gamma_{b}(\pi, \mathcal{D})$ with the cover-strict topology.
\end{abstract}

\section{INTRODUCTION}

The present article investigates the ideal and quotient structures of certain algebras of vector-valued functions. By using the theory of bundles of topological vector spaces, our results extend to more general algebras many of the results to be found in [1] and [3] regarding the structure of some ideals and quotients of $C(X)$, where $X$ is a completely regular Hausdorff space.

We will be concerned with certain subspaces and quotients of $\Gamma(\pi)$, the space of sections of the bundle of Banach spaces (i.e., Banach bundle) $\pi: \mathcal{E} \rightarrow X$, and in particular, we will investigate such structures when $\pi: \mathcal{E} \rightarrow X$ is a bundle of Banach algebras (i.e., Banach algebra bundle). (For details of the development of such bundles, and of bundles of topological vector spaces in general, we refer the reader to [5]; further elaboration can be found in [10], [12], [6], and [7].) The essentials are the following (they can be found, e.g., in [7]), but we repeat them here for convenience. Our paper is also related to the theory of approximation

Copyright 2016 by the Tusi Mathematical Research Group.

Received Aug. 10, 2015; Accepted Jan. 18, 2016.

*Corresponding author.

2010 Mathematics Subject Classification. Primary 46H25; Secondary 46H10.

Keywords. cover-strict topology, bundle of Banach spaces, bundle of Banach algebras. 